\title{
Compression of $\mathrm{CaTiO}_{3}$ and $\mathrm{CaGeO}_{3}$ perovskites
}

\author{
Nancy L. Ross ${ }^{1 *}$ and Ross J. Angel ${ }^{2}$ \\ ${ }^{1}$ Department of Geological Sciences, University College London, Gower Street, London, WC1E 6BT, U.K. \\ ${ }^{2}$ Bayerisches Geoinstitut, Universität Bayreuth, D95440 Bayreuth, Germany
}

\begin{abstract}
High-pressure single-crystal X-ray diffraction measurements of $\mathrm{CaTiO}_{3}$ and $\mathrm{CaGeO}_{3}$ perovskite have been carried out to 9.7 and $8.6 \mathrm{GPa}$, respectively, at room temperature. Fitting a third-order Birch-Murnaghan equation-of-state to the $P-V$ data yields values of $V_{0}=$ $223.764 \pm 0.017 \AA^{3}, K_{T, 0}=170.9 \pm 1.4 \mathrm{GPa}$, and $K^{\prime}=\partial K / \partial P=6.6 \pm 0.3$ for $\mathrm{CaTiO}_{3}$ and $V_{0}=206.490 \pm 0.017 \AA^{3}, K_{T, 0}=194.0 \pm 2.1 \mathrm{GPa}$, and $K^{\prime}=6.1 \pm 0.5$ for $\mathrm{CaGeO}_{3}$. A similar analysis of the axial compressibilities shows that the degree of anisotropic compression in both perovskites is less than $10 \%$. In $\mathrm{CaTiO}_{3}$ the $a$ and $b$ axes have similar compressibilities $\left(K_{\mathrm{a}}=168.7 \pm 2.1 \mathrm{GPa}, K_{\mathrm{b}}=168.3 \pm 1.9 \mathrm{GPa}\right)$ whereas the $c$ axis is the least compressible $\left(K_{\mathrm{c}}=175.3 \pm 1.5 \mathrm{GPa}\right)$. In $\mathrm{CaGeO}_{3}$, the $b$ axis $\left(K_{\mathrm{b}}=188 \pm 4 \mathrm{GPa}\right)$ and the $a$ axis $\left(K_{\mathrm{a}}=195 \pm 5 \mathrm{GPa}\right)$ are more compressible than the $c$ axis $\left(K_{\mathrm{c}}=204 \pm 3 \mathrm{GPa}\right)$. The variations with pressure of all axes show significant curvature with increasing pressure and have $K^{\prime}$ values ranging from $5.7 \pm 0.5$ to $7.0 \pm 0.4$ in $\mathrm{CaTiO}_{3}$ and $5.0 \pm 0.9$ to $6.9 \pm$ $1.2 \mathrm{in} \mathrm{CaGeO}_{3}$. No phase transition was detected. There is evidence, however, that in $\mathrm{CaGeO}_{3}$ the tetragonal to orthorhombic spontaneous strain decreases slightly with pressure which may indicate that a phase transition occurs at a pressure above $10 \mathrm{GPa}$. Elasticity trends of Ca-perovskites relating bulk modulus and molar volume are independent of both the degree of distortion from cubic symmetry and the symmetry of the structure.
\end{abstract}

\section{INTRODUCTION}

Many $\mathrm{ABO}_{3}$ compounds with the perovskite structure exhibit orthorhombic Pbnm symmetry under ambient conditions and are isotypic with $\mathrm{GdFeO}_{3}$. Of this group of perovskites, the $\mathrm{A}^{2+}-\mathrm{B}^{4+}$ perovskites are of particular interest to earth scientists because the lower-mantle inventory of $\mathrm{Mg}^{2+}$ and $\mathrm{Ca}^{2+}$ is believed to be contained in the silicate perovskites $\mathrm{MgSiO}_{3}$ and $\mathrm{CaSiO}_{3} \cdot \mathrm{MgSiO}_{3}$ is an orthorhombic $\mathrm{GdFeO}_{3}$-type perovskite at high pressures and temperatures (Funamori et al. 1996), whereas $\mathrm{CaSiO}_{3}$ has the ideal cubic structure with $P m \overline{3} m$ symmetry (Wang et al. 1996). The degree of distortion from the ideal cubic structure can be described by the tilting of the $\left[\mathrm{BO}_{6}\right]$ octahedra. Sasaki et al. (1983) showed that this tilting varies systematically with the size-ratio of the cations occupying the dodecahedral site $\left(\mathrm{A}^{2+}\right.$ cation) and octahedral site $\left(\mathrm{B}^{4+}\right.$ cation). The tilting of the octahedra may change with increasing pressure and/or temperature leading to phase transformations from orthorhombic symmetry.

Both the mineral perovskite, $\mathrm{CaTiO}_{3}$, and $\mathrm{CaGeO}_{3}$ with the perovskite structure (which is stable at pressures greater than $6 \mathrm{GPa}$ ) are $\mathrm{GdFeO}_{3}$-type perovskites (Sasaki et al. 1983, 1987). With increasing temperature, $\mathrm{CaTiO}_{3}$ undergoes a transition from the room temperature orthorhombic $(\mathrm{Pbnm})$ structure to a tetragonal $(\mathrm{I} / \mathrm{mcm})$ poly-

*E-mail: n.ross@ucl.ac.uk

0003-004X/99/0003-0277\$05.00 morph between 1373-1423 K followed by a transformation to the cubic $(P m \overline{3} m)$ aristotype at $1523 \mathrm{~K}$ (Redfern 1996). High-temperature X-ray powder diffraction experiments by Liu et al. (1991) suggested that $\mathrm{CaGeO}_{3}$ perovskite undergoes a phase transition at 520 $\mathrm{K}$ to a structure tentatively identified as having $\mathrm{Cmcm}$ symmetry, although high-temperature Raman scattering experiments showed no evidence for either a phase transition or soft-mode behavior between room temperature and $923 \mathrm{~K}$ (Durben et al. 1991).

In a high-pressure study, Xiong et al. (1986) reported that $\mathrm{CaTiO}_{3}$ transforms into a hexagonal phase at approximately $10 \mathrm{GPa}$ at room temperature on the basis of in situ X-ray diffraction, but Gillet et al. (1993) found no significant changes in the Raman spectrum of $\mathrm{CaTiO}_{3}$ perovskite to $21 \mathrm{GPa}$ at room temperature. High-pressure EXAFS spectra of $\mathrm{CaGeO}_{3}$ perovskite suggest that the structure becomes less distorted with increasing pressure, leading to a phase transition to a tetragonal structure at about $12 \mathrm{GPa}$ (Andrault and Poirier 1991). However, changes in the tilt angle and Ge-Ge distance are comparable to the experimental uncertainty. Lu and Hofmeister (1994), moreover, found no evidence of a phase transition to tetragonal symmetry from far-infrared spectra collected to $24.4 \mathrm{GPa}$. This study undertakes high-pressure, singlecrystal X-ray diffraction measurements of $\mathrm{CaTiO}_{3}$ and $\mathrm{CaGeO}_{3}$ perovskite to clarify whether any phase transitions occur with increasing pressure and to compare their 
TABLE 1. Unit-cell parameters of $\mathrm{CaTiO}_{3}$ and $\mathrm{CaGeO}_{3}$ perovskites

\begin{tabular}{|c|c|c|c|c|}
\hline $\begin{array}{c}\text { Pressure } \\
\text { (GPa) }\end{array}$ & $a(\AA)$ & $b(\AA)$ & $c(\AA)$ & $V\left(\AA^{3}\right)$ \\
\hline \multicolumn{5}{|c|}{$\mathrm{CaTiO}_{3}$} \\
\hline 0.001 & $5.38041(19)$ & $5.4432(2)$ & $7.6406(4)$ & $223.769(17)$ \\
\hline $1.157(5)$ & $5.36837(18)$ & $5.4311(2)$ & $7.6240(4)$ & $222.285(16)$ \\
\hline $1.960(5)$ & $5.36001(16)$ & $5.4231(2)$ & 7.6128(4) & $221.289(16)$ \\
\hline $2.913(5)$ & $5.35055(22)$ & $5.4137(3)$ & $7.6004(6)$ & $220.156(21)$ \\
\hline $4.232(6)$ & $5.33896(16)$ & $5.4020(2)$ & $7.5834(3)$ & $218.714(14)$ \\
\hline $5.303(8)$ & $5.32888(16)$ & $5.3918(2)$ & $7.5710(2)$ & $217.532(15)$ \\
\hline $5.782(9)$ & $5.32426(23)$ & $5.3875(3)$ & $7.5650(6)$ & $216.997(20)$ \\
\hline $6.940(8)$ & $5.31418(24)$ & $5.3778(2)$ & $7.5514(7)$ & $215.807(22)$ \\
\hline $7.685(9)$ & $5.30851(25)$ & $5.3720(3)$ & $7.5430(6)$ & $215.104(22)$ \\
\hline $8.848(9)$ & $5.29851(18)$ & $5.3626(2)$ & $7.5300(5)$ & 213.954(16) \\
\hline $9.700(9)$ & $5.29191(22)$ & $5.3565(3)$ & $7.5216(6)$ & $213.207(20)$ \\
\hline \multicolumn{5}{|c|}{$\mathrm{CaGeO}_{3}$} \\
\hline 0.0001 & $5.2614(3)$ & $5.2701(5)$ & $7.44677(19)$ & 206.484(18) \\
\hline $1.445(5)$ & $5.2498(3)$ & $5.2563(5)$ & $7.42964(22)$ & $205.016(19)$ \\
\hline $2.393(5)$ & $5.2406(3)$ & $5.2481(4)$ & $7.41831(20)$ & $204.025(17)$ \\
\hline $3.982(9)$ & $5.2280(4)$ & $5.2350(6)$ & $7.40114(30)$ & $202.557(24)$ \\
\hline $4.980(7)$ & $5.2206(3)$ & $5.2261(5)$ & $7.38977(19)$ & $201.617(17)$ \\
\hline $6.102(9)$ & $5.2120(3)$ & $5.2174(7)$ & $7.37826(25)$ & $200.639(24)$ \\
\hline $7.098(9)$ & $5.2050(2)$ & $5.2093(3)$ & $7.36758(14)$ & $199.769(12)$ \\
\hline $7.639(8)$ & $5.2011(3)$ & $5.2047(6)$ & $7.36203(23)$ & $199.291(20)$ \\
\hline $8.553(10)$ & $5.1948(3)$ & $5.1984(4)$ & $7.35355(18)$ & $198.577(15)$ \\
\hline
\end{tabular}

high-pressure behavior with their high-temperature behavior. In addition, present results provide insight into how changes in distortion within $\mathrm{GdFeO}_{3}$-type perovskites affect their elastic properties.

\section{EXPERIMENTAL METHODS}

Single crystals of $\mathrm{CaTiO}_{3}$ perovskite were kindly provided by E. Takayama-Muromachi (NIRIM, Tsukuba, Japan). Single crystals of $\mathrm{CaGeO}_{3}$ perovskite were synthesized at $10 \mathrm{GPa}$ and $1000{ }^{\circ} \mathrm{C}$ in a multi-anvil press at the Bayerisches Geoinstitut, Germany, using $\mathrm{CaGeO}_{3}$ wollastonite as the starting material.

High-pressure measurements were performed with BGI design diamond-anvil cells (Allan et al. 1996) using T301 steel gaskets. For each experiment, the perovskite crystal was loaded into the diamond-anvil cell together with a ruby chip for approximate pressure measurements and a quartz crystal as an internal diffraction pressure standard. A 4:1 mixture of methanol:ethanol was used as pressure medium. Diffraction experiments were performed on a Huber four-circle diffractometer on which the crystals gave sharp, well-defined peak profiles with FWHM approximately $0.08^{\circ}$ in $\omega$. Details of the instrument and the peak-centering algorithms are provided by Angel et al. (1997); the effect of crystal offsets and diffractometer aberrations were eliminated from refined peak positions by the eight-position centering method of King and Finger (1979). Unconstrained unit-cell parameters were determined from a least-squares fit to the corrected setting angles. No unit-cell angle exhibited any deviation greater than one estimated standard deviation (esd) from symmetry-constrained values. The values of symmetry-constrained, unit-cell parameters obtained by vector-leastsquares (Ralph and Finger 1982) are in Table 1. Pressures were determined from the unit-cell volumes of the quartz crystal in the diamond anvil cell, using the equation-ofstate reported by Angel et al. (1997). Equation-of-state parameters of the perovskites were obtained by a weighted-least-squares fit of the Birch-Murnaghan third-order equation-of-state (Birch 1947) to the pressure-volume data. Weights were calculated from the esd's in the unitcell volumes of the perovskites combined with the uncertainty in pressure corresponding to the esd's of the unit-cell volumes of the quartz pressure standard.

\section{Results AND Discussion}

The unit-cell parameters of both $\mathrm{CaTiO}_{3}$ and $\mathrm{CaGeO}_{3}$ are dimensionally close to being cubic under ambient conditions. The pseudo-cubic unit-cell parameters $\left(a_{\mathrm{C}}=\right.$ $a_{0} / \sqrt{ } 2, b_{\mathrm{C}}=b_{0} / \sqrt{ } 2$, and $c_{\mathrm{C}}=c_{0} / 2$ ) for $\mathrm{CaTiO}_{3}$ are: $a_{\mathrm{C}}=$ $3.805 \AA, b_{\mathrm{C}}=3.849 \AA, c_{\mathrm{C}}=3.820 \AA$, and for $\mathrm{CaGeO}_{3}$ are: $a_{\mathrm{C}}=3.720 \AA, b_{\mathrm{C}}=3.727 \AA, c_{\mathrm{C}}=3.723 \AA$. However, the structure refinements of Sasaki et al. (1983, 1987) show that the structures are considerably distorted from cubic due to rotation of the corner-linked octahedra. For example, the O2-O2-O2 angle that describes tilting in the $x$ - $y$ plane is $150.54(5)^{\circ}$ in $\mathrm{CaTiO}_{3}$ and $154.7(2)^{\circ}$ in $\mathrm{Ca}-$ $\mathrm{GeO}_{3}$, contrasting with $180^{\circ}$ for a cubic perovskite. Similarly, the value of the O2-O2-O2 angle that corresponds to tilting in the $x$ - $z$ plane is $101.77(4)^{\circ}$ and $100.2(1)^{\circ}$ for $\mathrm{CaTiO}_{3}$ and $\mathrm{CaGeO}_{3}$, respectively, compared to $90^{\circ}$ for a cubic perovskite. Thus, $\mathrm{CaTiO}_{3}$ is slightly more distorted from cubic symmetry than $\mathrm{CaGeO}_{3}$ perovskite, which is also reflected in their observed tolerance factors, $t_{\mathrm{obs}}$, of 0.986 and 0.989, respectively (Sasaki et al. 1983, 1987).

The metrical deviation of the orthorhombic perovskites from cubic or tetragonal symmetries can also be quantified as spontaneous strains, which can be calculated from the unit-cell parameters. The relevant strains for Pbnm perovskites correspond to possible cubic-tetragonal, $\mathrm{cu}-$ bic-orthorhombic and tetragonal-orthorhombic symmetry changes (Redfern 1996) with the corresponding strain going to zero at the phase transition. In $\mathrm{CaTiO}_{3}$, at room temperature and pressure, the cubic-tetragonal strain is lowest (0.0017) followed by the cubic-orthorhombic strain $(0.0075)$ followed by the tetragonal-orthorhombic strain (0.0116). With increasing temperature, the strain arising from the orthorhombic-tetragonal transition falls steeply to zero at approximately $1400 \mathrm{~K}$ and the orthorhombic- and tetragonal-type distortions of the cell, with respect to the cubic prototype, both extrapolate to zero at around $1520 \mathrm{~K}$ (Redfern 1996). By contrast, with increasing pressure we detect no significant change in any of these strains. There is, therefore, no evidence of a phase transition or any change in metrical distortion between 0 and $10 \mathrm{GPa}$ in $\mathrm{CaTiO}_{3}$ perovskite.

For $\mathrm{CaGeO}_{3}$ perovskite, the spontaneous strains are quite different from $\mathrm{CaTiO}_{3}$ perovskite. At room temperature and pressure the cubic-tetragonal strain is lowest (zero within the uncertainties) followed by the tetragonalorthorhombic strain (0.0017) and the cubic-orthorhombic strain (0.0126). Thus, $\mathrm{CaGeO}_{3}$ is much closer to being 
dimensionally tetragonal than $\mathrm{CaTiO}_{3}$ perovskite. Increased pressure produces no significant change in the cubic-orthorhombic strains. However, the tetragonal-orthorhombic strain and the cubic-tetragonal strain converge to a value of $\sim 0.0007$ at $8.553 \mathrm{GPa}$. Thus, although this data suggests that $\mathrm{CaGeO}_{3}$ perovskite is becoming slightly less distorted with increasing pressure, structural data is needed to confirm whether the octahedral tilts are changing significantly with increasing pressure. This structure, for example, may be approaching an orthorhombic to tetragonal transition at a pressure greater than 8.6 GPa, as suggested by the high-pressure EXAFS data of Andrault and Poirier (1991). Lu and Hofmeister (1994), however, found no evidence for a transition to a tetragonal structure. Infrared frequencies of $\mathrm{CaGeO}_{3}$ perovskite increase linearly with pressure and there are no discontinuous changes in either the number of peaks or the widths of the bands to $24.4 \mathrm{GPa}$.

Both the volumes and the individual unit-cell parameters show a smooth decrease with increasing pressure. Axial compressibilities of both perovskites were obtained by fitting a linear version of the Murnaghan EoS to the unit-cell parameter data from which the values of the unit-cell axis at room pressure $\left(d_{0}\right)$, the compressional modulus of the axis $\left(K_{\mathrm{d}}\right)$, and its pressure derivative $\left(K_{\mathrm{d}}^{\prime}\right)$ were obtained. For $\mathrm{CaTiO}_{3}: a_{0}=5.3803 \pm 0.0002 \AA, K_{\mathrm{a}}$ $=168.7 \pm 2.1 \mathrm{GPa}, K_{\mathrm{a}}^{\prime}=5.7 \pm 0.5 ; b_{0}=5.4433 \pm$ $0.0002 \AA, K_{\mathrm{b}}=168.3 \pm 1.9 \mathrm{GPa}, K_{\mathrm{b}}^{\prime}=7.0 \pm 0.4 ; c_{0}=$ $7.6405 \pm 0.0002 \AA, K_{\mathrm{c}}=175.3 \pm 1.5 \mathrm{GPa}, K_{\mathrm{c}}^{\prime}=6.6 \pm$ 0.3. For $\mathrm{CaGeO}_{3}: a_{0}=5.2617 \pm 0.0004 \AA, K_{\mathrm{a}}=195 \pm$ $5 \mathrm{GPa}, K_{\mathrm{a}}^{\prime}=6.9 \pm 1.2 ; b_{0}=5.2699 \pm 0.0003 \AA, K_{\mathrm{b}}=$ $188 \pm 4 \mathrm{GPa}, K_{\mathrm{b}}^{\prime}=5.0 \pm 0.9 ;$ and $c_{0}=7.4468 \pm 0.0003$ $\AA, K_{\mathrm{c}}=204 \pm 3 \mathrm{GPa}, K_{\mathrm{c}}^{\prime}=5.3 \pm 0.6$. It is clear that in both $\mathrm{CaTiO}_{3}$ and $\mathrm{CaGeO}_{3}$ the compressibilities of the $a$ and $b$ axes are similar and the $c$ axis is the least compressible. In $\mathrm{CaGeO}_{3}$ the $b$ axis converges toward $a$ with increasing pressure leading to a reduction in the tetragonal-orthorhombic strain, as noted. The total amount of anisotropy in the axial compressibilities is less than $10 \%$ in both perovskites.

A third-order Birch-Murnaghan equation-of-state was fit to the $P$-V data (Fig. 1) for both perovskites. $\mathrm{CaTiO}_{3}$ reveals $V_{0}=223.764 \pm 0.017 \AA^{3}, K_{T, 0}=170.9 \pm 1.4 \mathrm{GPa}$, and $K^{\prime}=6.6 \pm 0.3$ with a $\chi_{\mathrm{w}}^{2}=1.4$ and $\Delta P_{\max }=\mid P_{\mathrm{obs}}-$ $\left.P_{\text {cald }}\right|_{\max }=0.03 \mathrm{GPa}$. For $\mathrm{CaGeO}_{3}$, this analysis yields $V_{0}$ $=206.490 \pm 0.017 \AA^{3}, K_{T, 0}=194.0 \pm 2.1 \mathrm{GPa}$, and $K^{\prime}$ $=6.1 \pm 0.5$ with a $\chi_{\mathrm{w}}^{2}=1.1$ and $\Delta P_{\max }=0.03 \mathrm{GPa}$. The $\chi_{w}^{2}$ values indicate that the esd's of the unit-cell volumes of the perovskites and pressure calibrants are realistic estimates of the measurement precision. Both perovskites have $K^{\prime}$ values significantly greater than 4 . If $K^{\prime}$ is constrained to 4 , significantly worse fits to the data were obtained. If $K^{\prime}=4$ for $\mathrm{CaTiO}_{3}, x_{\mathrm{w}}^{2}$ increases to 11.3 and $\Delta P_{\max }=0.12 \mathrm{GPa}$, and for $\mathrm{CaGeO}_{3}, \chi_{\mathrm{w}}^{2}$ increases to 3.6 and $\Delta P_{\max }=0.06 \mathrm{GPa}$.

The value of the isothermal bulk modulus determined for $\mathrm{CaTiO}_{3}$ as $170.9 \pm 1.4 \mathrm{GPa}$ is in excellent agreement with values of the adiabatic bulk moduli $\left(K_{\mathrm{s}}\right)$ determined

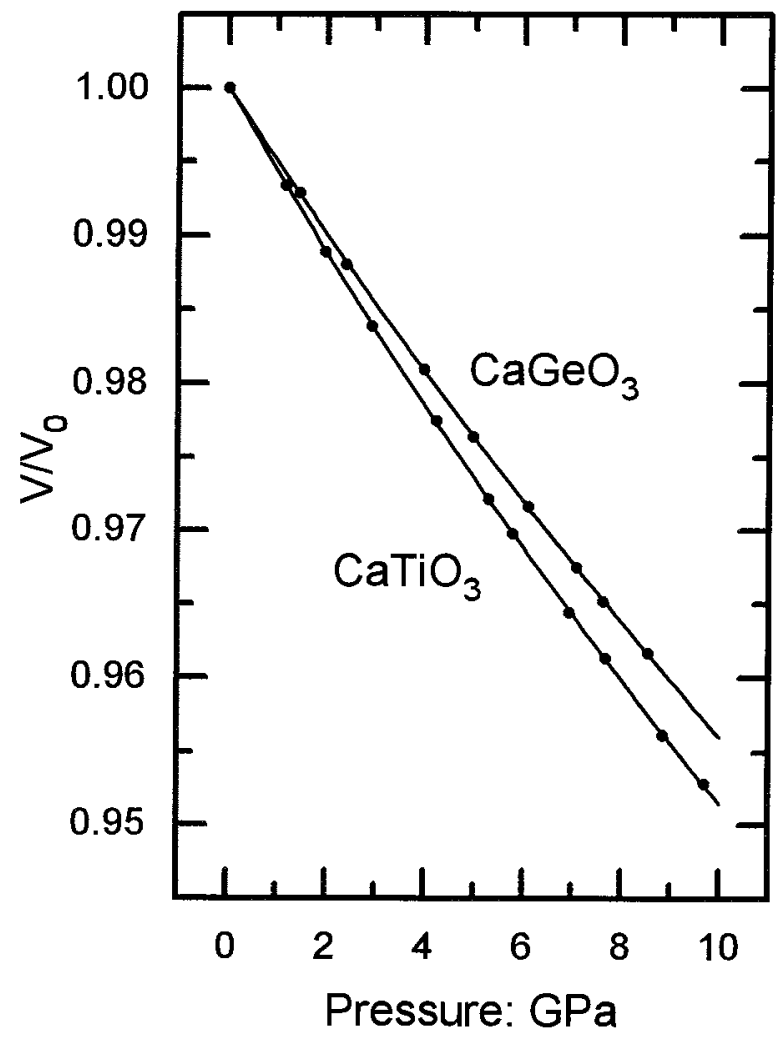

Figure 1. Variation of $V / V_{0}$ of $\mathrm{CaTiO}_{3}$ and $\mathrm{CaGeO}_{3}$ perovskites as a function of pressure. The size of the symbols exceeds the experimental esd. The lines are the Birch-Murnaghan equations-of-state fitted to the data.

from ultrasonic measurements: $177 \pm 3 \mathrm{GPa}$ (Liebermann et al. 1977), $177 \pm 3 \mathrm{GPa}$ (Fischer et al. 1993), $175 \pm 1$ GPa (Sinelnikov et al. 1998), and $175 \mathrm{GPa}$ (Kung and Rigden 1998), when allowance is made for the conversion between adiabatic and isothermal values $\left(K_{\mathrm{S}}=K_{\mathrm{T}}(1+\right.$ $\alpha \gamma T) \sim 1.01-1.02 K_{T}$ at $\left.300 \mathrm{~K}\right)$. The value of $K^{\prime}$ is consistent with $\left(\mathrm{d} K_{\mathrm{S}} / \mathrm{d} P\right)=5.78$ measured by Kung and Rigden (1998) and the reanalysis of the data of Fischer et al. (1993) by Kung and Rigden (1998), which yields $\left(\mathrm{d} K_{\mathrm{S}} /\right.$ $\mathrm{d} P)=6.0 \pm 0.8$. There is only one ultrasonic study of $\mathrm{CaGeO}_{3}$ perovskite from which $K_{\mathrm{S}}=198 \pm 15 \mathrm{GPa}$ (Liebermann et al. 1977) is in agreement with this study's determination of $K_{T, 0}$.

The relationship between bulk modulus and specific volume $\left(V_{\mathrm{m}}\right)$ is well established for simple structure types in the absence of phase transitions, and the product $K V_{\mathrm{m}}$ has been shown to be a constant for oxides with a given crystal structure and valence product (e.g., Anderson and Anderson 1970). In the case of perovskites, it is possible that structural phase transitions could influence the elastic properties, even at pressures and temperatures far removed from the phase transitions. However, previous analyses of ultrasonic data of various perovskites (e.g., Liebermann et al. 1977; Bass 1984) found no deviations in trends of bulk moduli and bulk sound velocities with mo- 


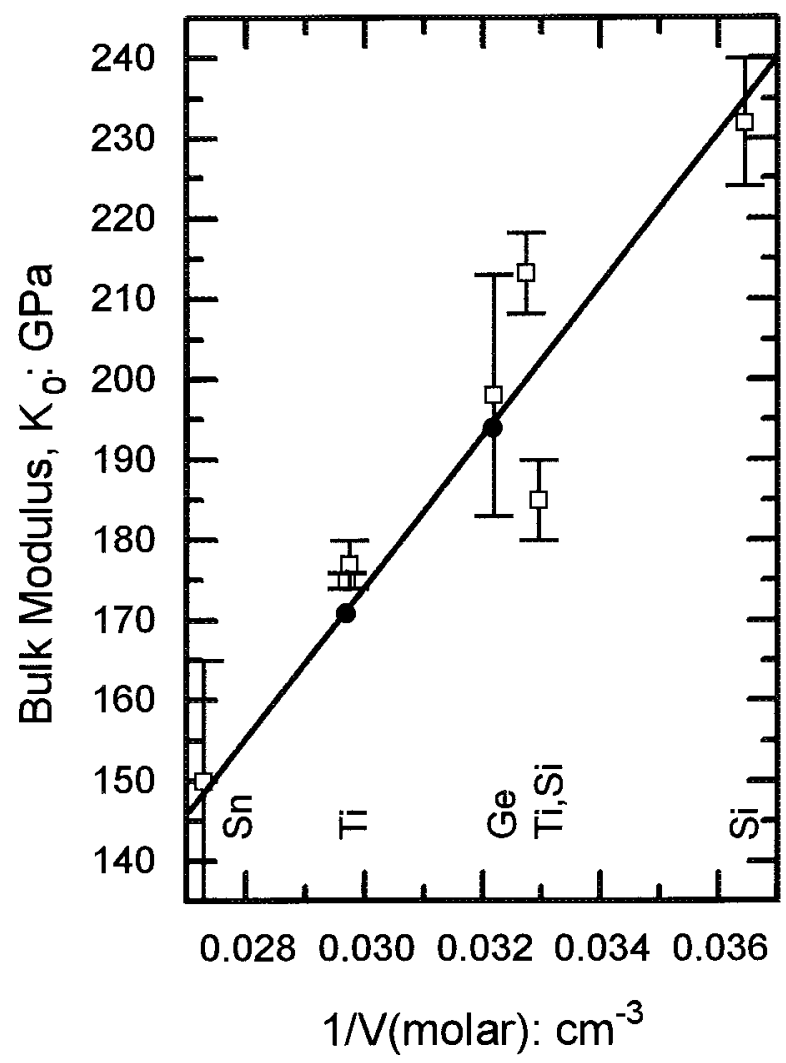

Figure 2. Bulk moduli of Ca-perovskites plotted against the inverse of the molar volume. The $\mathrm{B}$ cation is indicated on the plot. Solid dots $=\mathrm{CaTiO}_{3}$ and $\mathrm{CaGeO}_{3}$ from this study. Error bars $=\mathrm{CaSnO}_{3}$ (Liebermann et al. 1977); $\mathrm{CaSiO}_{3}$ (Wang et al. 1996); $\mathrm{Ca}\left(\mathrm{Si}_{0.5} \mathrm{Ti}_{0.5}\right) \mathrm{O}_{3}$ Sinelnikov et al. (1998) and Angel et al. (unpublished manuscript); $\mathrm{CaTiO}_{3}$ Fischer et al. (1993) and Sinelnikov et al. (1998); $\mathrm{CaGeO}_{3}$ Liebermann et al. (1977).

lar volume within the uncertainties of the data then available. More recently, Fischer et al. (1993) concluded that crystal structure type does have a significant effect on elasticity, whereas consistent trends can be defined within orthorhombic perovskites. The availability of more precise data, such as that presented here, provides an opportunity to test such proposed trends to a higher level of accuracy. Figure 2 indicates that within the calcium perovskites, most data are consistent with the trend $K_{0}=$ $9277 / V$ (molar) - $104.4 \mathrm{GPa}$ defined by the data for $\mathrm{CaGeO}_{3}$ and $\mathrm{CaTiO}_{3}$. Such a trend predicts $K_{0}=233 \mathrm{GPa}$ for $\mathrm{CaSiO}_{3}$ perovskite, in good agreement with $K_{T}=232$ $\pm 8 \mathrm{GPa}$ determined by Wang et al. (1996) from in situ $\mathrm{X}$-ray diffraction measurements. The recent lower estimate of $212 \pm 7 \mathrm{GPa}$ for $\mathrm{CaSiO}_{3}$ by Sinelnikov et al. (1998) arises from their use of a value of $K_{\mathrm{S}}$ for $\mathrm{Ca}\left(\mathrm{Ti}_{0.5} \mathrm{Si}_{0.5}\right) \mathrm{O}_{3}$ of $188 \pm 1 \mathrm{GPa}$, which falls significantly below the general trend. However, a recent determination of the EoS by Angel et al. (unpublished data) suggests a $K_{0} \sim 213 \mathrm{GPa}$, significantly higher than the general trend (Fig. 2). With this one possible exception, the results from the X-ray diffraction study confirm previous conclusions of Liebermann et al. (1977) and Bass (1984) that the trend relating bulk modulus to molar volume is independent of both the degree of distortion from cubic symmetry as well as the symmetry of the perovskite structure.

\section{ACKnowledgments}

E. Takayama-Muromachi is gratefully acknowledged for synthesizing $\mathrm{CaTiO}_{3}$ perovskite. High-pressure synthesis of $\mathrm{CaGeO}_{3}$ perovskite and high-pressure single-crystal X-ray diffraction experiments were performed at the Bayerisches Geoinstitut, Bayreuth, Germany under the EC Human Capital and Mobility-Access to Large Scale Facilities programme (contract no. ERBCHGECT940053 to D.C. Rubie).

\section{REFERENCES CITED}

Allan, D.R., Militech, R., and Angel, R.J. (1996) A diamond-anvil cell for single-crystal X-ray diffraction studies to pressures in excess of 10 GPa. Reviews of Scientific Instruments, 67, 840-842.

Anderson, D.L. and Anderson, O.L. (1970) Bulk modulus-volume relationship for oxides. Journal of Geophysical Research, 75, 3494-3500.

Andrault, D. and Poirier, J.P. (1991) Evolution of the distortion of perovskites under pressure: An EXAFS study of $\mathrm{BaZrO}_{3}, \mathrm{SrZrO}_{3}$, and Ca$\mathrm{GeO}_{3}$. Physics and Chemistry of Minerals, 18, 91-105.

Angel, R.J., Allan, D.R., Militech, R., and Finger, L.W. (1997) The use of quartz as an internal pressure standard in high-pressure crystallography. Journal of Applied Crystallography, 30, 461-466.

Bass, J.D. (1984) Elasticity of single-crystal $\mathrm{SmAlO}_{3}, \mathrm{GdAlO}_{3}$, and $\mathrm{ScAlO}_{3}$ perovskites. Physics of the Earth and Planetary Interiors, 36, $145-156$.

Birch, F. (1947) Finite elastic strain of cubic crystals. Physical Review, $71,809-824$

Durben, D.J., Wolf, G.H., and McMillan, P.F. (1991) Raman scattering study of the high-temperature vibrational properties and stability of $\mathrm{CaGeO}_{3}$ perovskite. Physics and Chemistry of Minerals, 18, 215-223.

Fischer, G.F., Wang, Z., and Karato, S. (1993) Elasticity of $\mathrm{CaTiO}_{3}, \mathrm{SrTiO}_{3}$, and $\mathrm{BaTiO}_{3}$ perovskites up to $3.0 \mathrm{GPa}$ : the effect of crystallographic structure. Physics and Chemistry of Minerals, 20, 97-103.

Funamori, N., Yagi, T., Utsumi, W., Kondo, T., and Uchida, T. (1996). Thermoelastic properties of $\mathrm{MgSiO}_{3}$ perovskite determined by in situ X-ray observations up to $30 \mathrm{GPa}$ and $2000 \mathrm{~K}$. Journal of Geophysical Research, 101, 8257-8269.

Gillet, P., Guyot, F., Price, G.D., Tournerie, B., and Le Cleach, A. (1993) Phase changes and thermodynamic properties of $\mathrm{CaTiO}_{3}$ : spectroscopic data, vibrational modeling, and some insights on the properties of $\mathrm{MgSiO}_{3}$ perovskite. Physics and Chemistry of Minerals, 20, 159-170.

King, H. and Finger, L.W. (1979) Diffracted beam centering and its application to high-pressure crystallography. Journal of Applied Crystallography, 12, 374-378.

Kung, J. and Rigden, S. (1998) Oxide perovskites: pressure derivatives of the bulk and shear moduli. Physics and Chemistry of Minerals, accepted.

Liebermann, R.C., Jones, L.A., and Ringwood, A.E. (1977) Elasticity of aluminate, titanate, stannate, and germanate compounds with the perovskite structure. Physics of the Earth and Planetary Interiors, 14, 165-178.

Liu, X., Wang, Y., Liebermann, R.C., Maniar, P.D., and Navrotsky, A. (1991) Phase transition in $\mathrm{CaGeO}_{3}$ perovskite: evidence from X-ray powder diffraction, thermal expansion, and heat capacity. Physics and Chemistry of Minerals, 18, 224-230.

$\mathrm{Lu}$, R. and Hofmeister, A.M. (1994) Infrared spectroscopy of $\mathrm{CaGeO}_{3}$ perovskite to $24 \mathrm{GPa}$ and thermodynamic implications. Physics and Chemistry of Minerals, 78-84.

Ralph, R.L. and Finger, L.W. (1982) A computer program for refinement of crystal orientation matrix and lattice constants from diffractometer data with lattice constraints. Journal of Applied Crystallography, 15, 537-539.

Redfern, S.A.T. (1996) High-temperature structural phase transitions in perovskite $\left(\mathrm{CaTiO}_{3}\right)$. Journal of Physics: Condensed Matter, 8, 82678275.

Sasaki, S., Prewitt, C.T., and Liebermann, R.C. (1983) The crystal struc- 
ture of $\mathrm{CaGeO}_{3}$ perovskite and the crystal chemistry of $\mathrm{GdFeO}_{3}$-type perovskites. American Mineralogist, 68, 1189-1198.

Sasaki, S., Prewitt, C.T., Bass, J.D., and Shultze, W.A. (1987) Orthorhombic perovskite $\mathrm{CaTiO}_{3}$ and $\mathrm{CdTiO}_{3}$ : structure and space group. Acta Crystallographica (vol C43), 1668-1674.

Sinelnikov, Y.D., Chen, G., and Liebermann, R.C. (1998) Elasticity of $\mathrm{CaTiO}_{3}-\mathrm{CaSiO}_{3}$ perovskites. Physics and Chemistry of Minerals, in press.

Wang, Y., Weidner, D.J., and Guyot, F. (1996) Thermal equation-of-state of $\mathrm{CaSiO}_{3}$ perovskite. Journal of Geophysical Research: B, 101, 661672.

Xiong, D.H., Ming, L.C., and Manghnani, M.H. (1986) High-pressure phase transformations and isothermal compression in $\mathrm{CaTiO}_{3}$ (perovskite). Physics of the Earth and Planetary Interiors, 43, 244-252.

ManusCRipt ReCeived June 23, 1998

Manuscript acCepted September 22, 1998

Paper handled by Robert M. Hazen 